\title{
User Interfaces for Creativity Support Tools
}

\author{
Ben Shneiderman \\ Human-Computer Interaction Laboratory, \\ Department of Computer Science and Institute for Systems Research, \\ University of Maryland, College Park, MD 20742, USA
}

\begin{abstract}
A challenge for human-computer interaction researchers and user interface designers is to construct information technologies that support creativity. This ambitious goal can be attained by building on an adequate understanding of creative processes. This paper expands on the four-phase genex framework for generating excellence [1]. Within this integrated framework, this paper proposes eight activities that require human-computer interaction research and advanced user interface design.
\end{abstract}

\section{INTRODUCTION}

Information technologics that allow more pcople to be more creative more of the time will have profound effects. Education will shift from acquiring facts, studying existing knowledge, or even developing critical thinking, to an emphasis on creating novel artifacts, insights, or performances. Medicine's shift from applying standard treatments to tailoring treatments for each patient, reflects the trend to personalization that is already ascendant in marketing and media. Expectations of teachers, lawyers, and designers are likely to rise as creativity is expected on more occasions from more people.

This paper reviews the genex framework which is a fourphase integrated framework to support creativity [1]. Then it describes eight activities that need powerful user interfaces to support creative work. This list implies a research agenda for human-computer interaction theoreticians, designers, software engineers, and evaluators. It seems necessary to apologize in advance for the hubris or arrogance of proposing technology to aid human creativity. A critic might scowl that creativity is inherently human and no computer could or should be brought into the process. But technology has always been part of the creative process, whether in Leonardo's paint and canvas or Pasteur's microscopes and beakers. Supportive technologies can become the potter's wheel and mandolin of creativity -- opening new media of expression and enabling compelling performances. Secondly, I seek tools that support human creativity and strongly rebel at the

Permission to make digital or hard copies of all or part of this work for personal or classroom use is granted without fee provided that copies are not made or distributed for profit or conmercial advantage and that copies bear this notice and the full citation on the first page. To copy otherwise, to republish, to post on servers or to redistribute to lists. requires prior specific permission and/or a tee.

Creativity \& Cognition 99 Loughborough UK

Copyright ACM 1999 1-58113-078-3/99/10...\$5.00 suggestion that machines are or can ever be creative. Computers have no more intelligence or creativity than a wooden pencil. People are creative and they have always used advanced technology to raise their potentials.

My expectations are largely positive, but there are many problems, costs, and dangers in anything as ambitious as a tool and framework to support creativity. An obvious concem is that many pcople may not want to be more creative. Many cultures encourage respect for the past and discourage disruptive innovations. Promoting widespread creativity raises expectations that may change employment patterns, educational systems, and community norms. Introducing computer supports for creativity may produce greater social inequality as it raises the costs for those who wish to participate. Finally, these tools may be used equally by those who have positive and noble goals as well as by dictators or criminals who seek to dominate, destroy, or plunder.

These fears are appropriate and reasonable cautions must be taken, but support for innovation could lead to more rapid positive changes to our world. Widespread access to creativity support tools could help with major problems such as environmental destruction, over population, poor medical care, oppression, and illiteracy. It could contribute to improvements in agriculture, transportation, housing, communication, and other noble human endeavors.

\section{GENEX: A FOUR-PHASE FRAMEWORK FOR GENERATING EXCELLENCE}

The genex framework [1] was a first attempt to build on Csikszentmihalyi's model [2] of creativity, by supporting access to the domain and consultation with the field. The name genex (generator of excellence) was chosen to echo Vannevar Bush's memex (memory extender). The genex framework has four phases:

- Collect: learn from previous works stored in digital libraries, the web, etc.

- Relate: consult with peers and mentors at early, middle and late stages

- Create: explore, compose, evaluate possible solutions

- Donate: disseminate the results and contribute to the digital libraries 
These four phases are not a linear path, creative work may require returning to earlier phases and much iteration. For example, digital libraries may be useful at every phase and discussion with peer and mentors may take place repeatedly during the development of an idea. This fourphase framework has much in common with previous characterizations and methodologies $[3,4]$, but there are important distinctions. Couger [5] reviews 22 "creative problem solving methodologies" and offers his own plan with five phases:

- Opportunity, delineation, problem definition

- Compiling relevant information

- Generating ideas

- Evaluating, prioritizing ideas

- Developing an implementation plan

The goal of genex framework is to suggest improvements for existing web-based services [6] and personal computer software tools. By reducing the distraction caused by poorly-designed user interfaces, users's attention can be devoted to the task. In an effective design for a genex, the boundaries between applications and the burdens of data conversions would disappear. Data representations and available functions would be in harmony with problemsolving strategies. Then users could concentrate on the four phases of being creative:collecting, relating, creating, and donating.

\section{INTEGRATING CREATIVE ACTIVITIES}

The genex framework calls for integrated creativity support tools. Some of these tools already exist, but could be enhanced to better support creativity. However, the main challenge for designers is to ensure smooth integration across these novel tools and with existing tools such as word processors, presentation graphics, email, databases, spreadsheets, and web browsers [7].

Smoother coordination across windows and better integration of tools seem possible. Just as word processors expanded to include images, tables, equations, and more, the next generation of software is likely to integrate additional features. Some aspects of the integration can be accomplished by creating compatible data types and file formats (possibly standardized objects). A second aspect of integration has to do with compatible actions and consistent terminology, such as cut-copy-paste or opensave-close. Higher levels of actions that are closer to the task domain might be candidates such as annotate-consultrevise or collect-explore-visualize. A third aspect of integration is the smooth coordination across windows [8]. For example, if users see an unfamiliar term they should be able to click on it and get an English definition, a French translation, or a medical dictionary report, all in a predictable screen location. Similarly, if users find a personal name in a document they should be able to get a biography, email address, or contact information. Other coordinations include synchronized scrolling of related documents to facilitate comparisons and hierarchical browsing of a table of contents and a chapter to enable easy navigation of large resources.

These improved user interfaces will benefit many users for many tasks, but my concern is specifically to develop creativity support within the genex framework (Figure 1).

\section{Genex Phase}

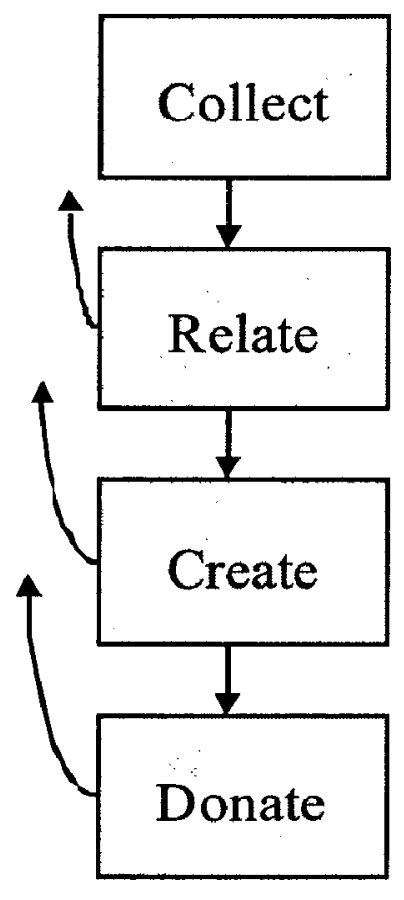

Primary Activity (but can be used at any phase)

$$
\begin{aligned}
& \text { Searching and browsing digital libraries } \\
& \text { Visualizing data and processes }
\end{aligned}
$$

\author{
Consulting with peers and mentors
}

Figure 1: Genex phases and their related primary activities

\section{Disseminating results}

\begin{abstract}
Thinking by free associations
Exploring solutions - What if tools

Composing artifacts and performances

Reviewing and replaying session histories
\end{abstract}


Genex's integrated creativity support tools should offer at least the eight activities described below. Figure 1 indicates how these activities are primarily related to the genex phases, but these activities could take place during any phase. For example searching of digital libraries is primarily associated with the collect phase, but searching may occur in order to find consultants or to decide on candidate communities for presenting of results. Visualizing objects and processes is the activity that seems most prolific and could appear in every genex phase.

\section{Searching and Browsing Digital Libraries}

Existing web search engines and digital library interfaces do an impressive job, but refinements to support creativity are possible. Users who want to steer the search to resources about previous work will want more control over the relevance ranking and over the presentation of the results [9]. For example, it should be easy for users to restrict a legal search to recent New York, New Jersey or Connecticut court cases on tenants rights for heating. The result set should be grouped by claims (civil rights, contract violation, etc.) and ordered by date with colorcoded stars indicating the strength of the results for or against tenants. Extraction of plaintiff's or defendant's lawyers should also be convenient. This may sound like a tall order, but detailed search request specification while maintaining comprehensibility is possible. Since searching is part of a larger creative process the result set should be easy to save into a spreadsheet for further manipulation, or included in an email note for consultation, or pasted into a notebook for later referral.

In addition to searching, many innovators will want rapid browsing to more effectively support exploration, similar to what many people do in bookstores or libraries. Web sites with high branching factors (many links per page) support exploration by making what is available more visible. Yahoo's (www.yahoo.com) 100+ links on the home page are helpful in getting an idea of what is available and what is not. Browsing textual menus supports exploration when specific terms or concepts are not known. Imagine the complex request implied by this question: which strains of bacteria are causing recent flu symptoms for senior citizens in the Mid-Atlantic states? No specific search string could produce appropriate results, but browsing through search results may lead to exploration of viral infections, variant symptoms, other diseases, other locations, earlier epidemics, etc.

Information derived from searching digital libraries may provide the right results, but there are still many concerns about whether the information is accurate, complete, and up-to-date. Furthermore, online information may only be a fraction of the total information, and the search engines may search only a fraction of the online information.

\section{Consulting with Peers and Mentors}

Email, listservs, newsgroups, and threaded discussions are excellent asynchronous tools for inquiries about previous work and for discussing new ideas. The capacity to find people who have a shared interest and are at work on similar problems is one of the greatest gifts of the internet. Synchronous tools, such as phone calls, videoconferencing (for example CUSeeMe [10]) and software sharing (for example, Netmeeting), enrich the possibilities for early, middle, and late stage consultations [11].

For early stage consultations, innovators are likely to search widely, finding diverse information resources and broad communities. At this preparatory stage, innovators are forming questions, finding out about current workers who might be collaborators and choosing directions. During middle stage consultations, the tasks are to propose potential solution strategies and develop evaluation methods. As solutions are created and refined, late stage consultations are directed at confirming the innovation, refining it to accommodate criticisms, and then disseminating it to appropriate parties [12]

Consultations might involve continuing dialogs by email, phone calls, or personal visits. While these may often be collegial and friendly, some discussions might be tense since other workers may be competitors who are pursuing similar goals. Either party might withhold some of their knowledge or ideas, or probe the other to gauge their intentions. Non-disclosure or non-competitive agreements might be discussed or signed as part of a corporate consultation. University researchers are typically open, but the competition for solution of important problems and battles to promote reputations can suppress discussion.

These concerns greatly influence the design and use of technologies, since the appropriate balance of privacy protection and easy access to information is vital. Researcher's early notes and explorations need to be kept private, but claimed breakthroughs need rapid and broad dissemination. Appropriate credits for articles, patents, or software products are often the source of conflicts, so improved record keeping of the consultations that contribute to an innovation could resolve some questions and maybe encourage more cooperation.

\section{Visualizing Data and Processes}

Many creative people are likely to propose visual approaches, because they appreciate the thoroughness and rapidity with which alternatives can be reviewed visually. The field of pharmaceutical drug discovery involves review of thousands of compounds for their efficacy, shelf life, solubility, acidity, cost, allergic reactions, interactions with other medications, etc. Scrolling lists in spreadsheets are useful, but two-dimensional visual presentations with color and size coding are proving to be enormous benefits for this task. Early work on a FilmFinder [13] led to a successful commercial product called Spotfire (http://www.spotfire.com) (Figure 2). A wide range of 1-, 2-, 3- and multi-dimensional visualizations plus temporal, tree and network presentations have been proposed [14]. 


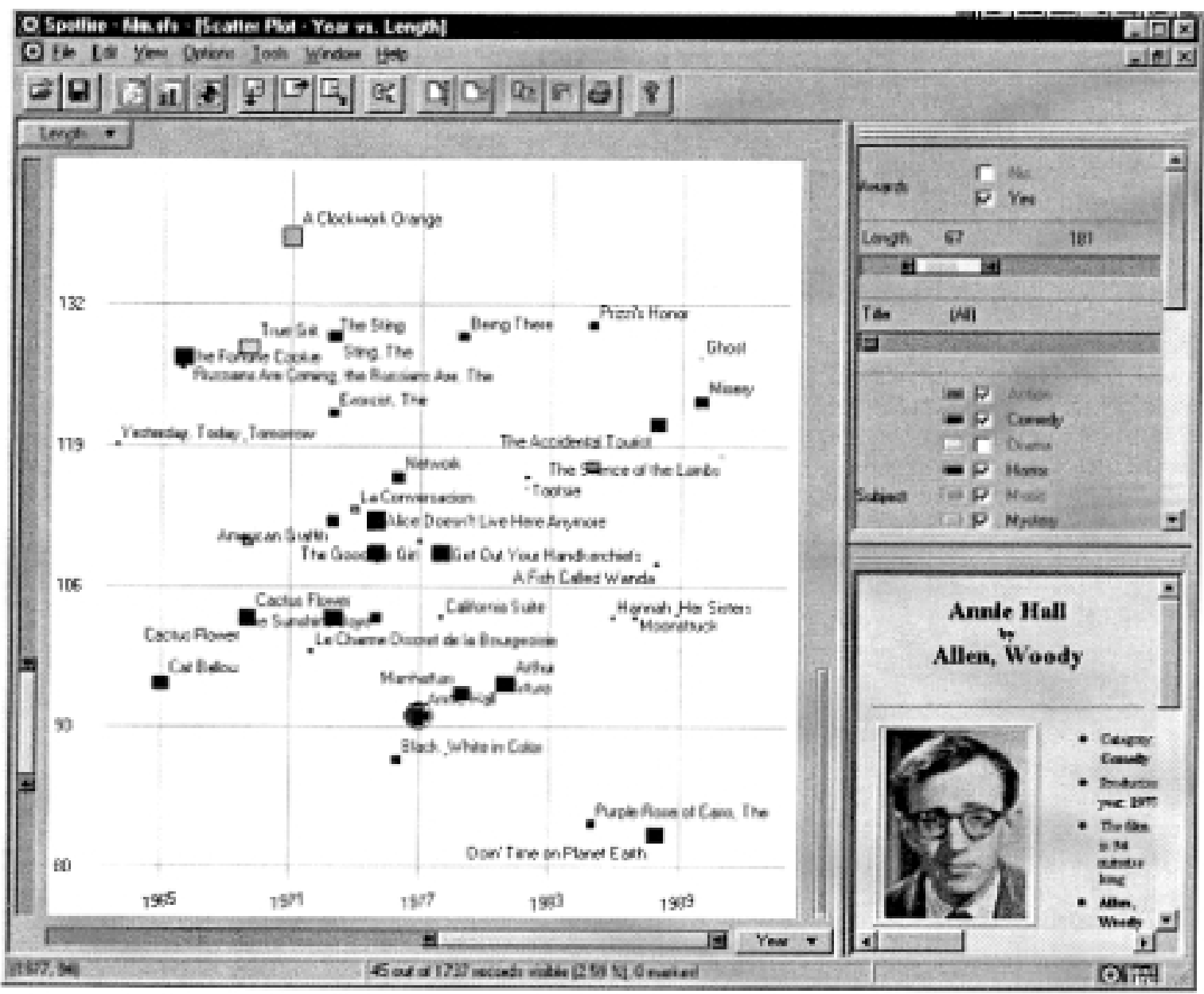

Figure 2

Visualization of digital library contents, financial data, product sales, legal information, medical histories [15], etc. is growing, but to increase its benefits to creativity requires smooth integration. The results of a web or database search should be easily imported (for example by cut and paste) into a visualization. Then users should be able to filter the data appropriately and adjust the visualization features, such as $x, y$-axes, color, size, or other codings. Then when an interesting group of items is found, users should be able to select them and paste them into a spreadsheet or statistics package for further processing. Then the visualization and processed items should be embeddable in a written report, slide presentation, or email note. Email recipients should be able to manipulate the visualization or report still further [16].

\section{Thinking by Free Associations}

Some software designers seek to liberate the mind by making free associations to related concepts. This Gestalt psychology approach [17] has led to innovative software that is meant to facilitate association of ideas by presenting related concepts. IdeaFisher (http://www.ideafisher.com) claims "to create a new method of generating ideas in a way similar to the workings of the human mind...by associations, or links...a thought like food leads to associations such as apple, cooking, cafe, washing dishes, and so on. When a creative new idea is born, it usually consists of associations linked together in a way that has not been thought of before. This is the heart of IdeaFisher technology-- a dictionary of associations." Users apparently enjoy using IdeaFisher but its benefits are still being understood [18].

Computerized thesauri may also be helpful textual exploration tools since varied associations such as synonyms, antonyms, homonyms, rhymes, or even anagrams can be retrieved rapidly. Alternatively, random word presentations are also proposed as a method for stimulating fresh thoughts and breaking through creative blocks.Other products enable users to create visual representations of relationships among words or concepts, including MindManager (http://www.mindman.com/) which allows users to "create relationships among information easily... identify relationships between items or branches." The Axon Idea Processor is promoted as "a sketchpad for visualizing and organizing ideas" that "exploits visual attributes such as: color, shape, size, scale, position, depth, link, icon, etc. Visual cues facilitate recall, association, and discovery. 


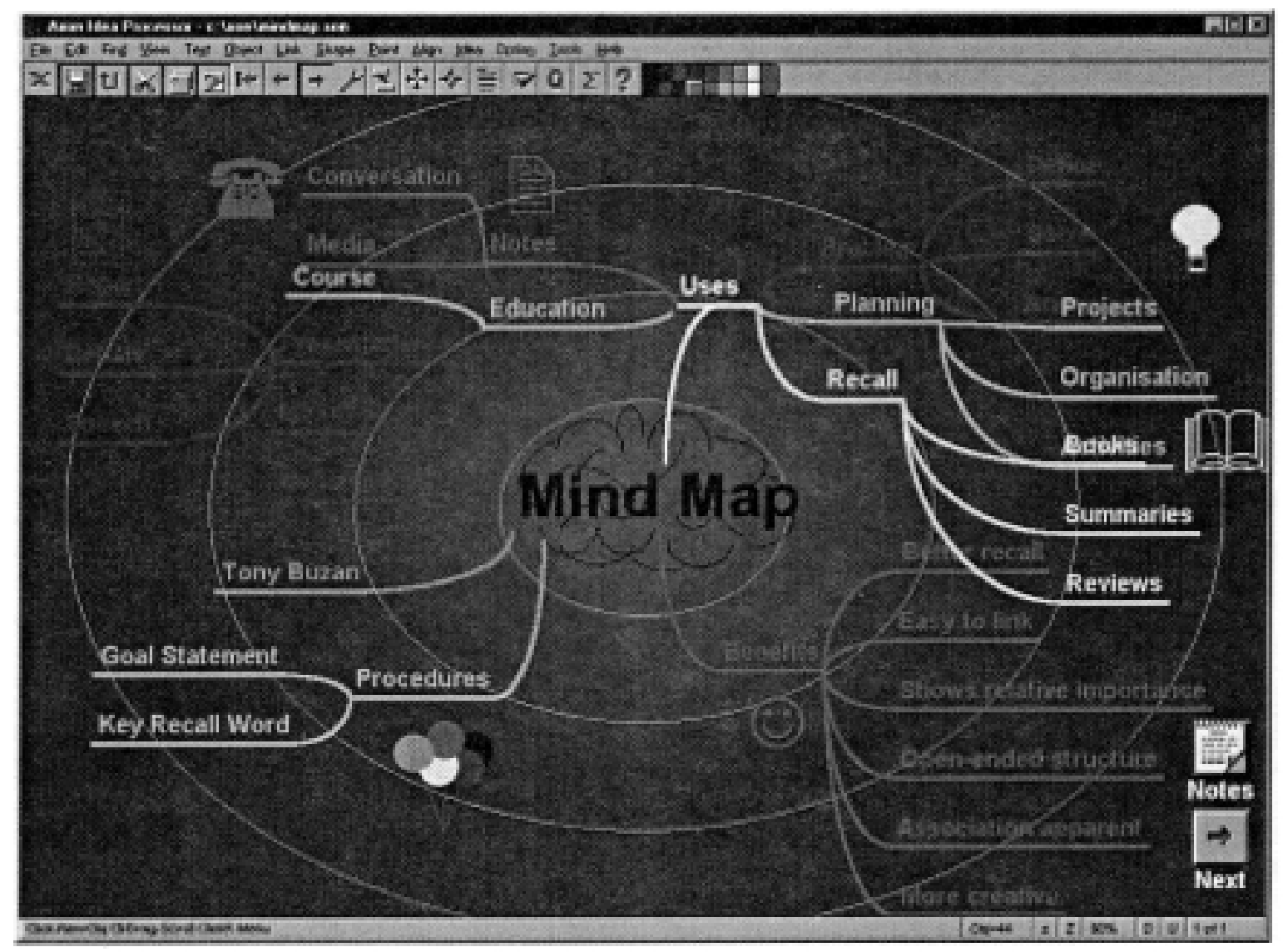

Figure 3

Mindmaps, concept maps, semantic networks and other drawing strategies may be helpful to some people. Appropriate visual presentations, such as the periodic table of elements, help in problem solving because they compactly present substantial information and show important relationships. Geographic maps, architect's drawings, and electronic circuit diagrams are other examples of the great power of visual presentations for learning and problem solving. The gift of the computer is the capacity to quickly create and easily manipulate such diagrams. Penciled diagrams and sketches have a similar capacity to invite discussion, exploration, and revision.

The genex framework reminds us that such textual lists and diagrams should be easily shared with others, annotatable, linkable, and searchable. It should be possible to import and export from these programs so that related tools can be employed, for example, to translate terminology in a diagram into a foreign language or to link diagram nodes to web sites.

\section{Exploring Solutions - What if? Tools}

A large family of software tools already support methodical exploration of solutions [19]. Electronic spreadsheets were quickly described as "what if" tools that allowed business planners and analysts to quickly try out a variety of scenarios. Such explorations were possible with paper and pencil, but they were a hundred times faster with electronic spreadsheets. Similarly, flight simulators, traffic flow models, weather models, and thousands of scientific, engineering, economic and other modeling tools allow users to explore alternatives safely, economically, and rapidly. Simulators have been largely custom built, but simulation building software is growing from domain specific to broader coverage. These simulation models are also becoming more richly featured to support explanatory text, collaborative usage, history keeping, and more [20]. A popular class of home computer software include simulation games such as SimCity, that enable users to try out urban planning scenarios. Also popular with personal computer users are flight, driving, and battle simulators, that provide various levels of realistic experiences. These applications are enjoyable and also educational, however they are largely independent.

The genex framework reminds us of the importance of integration to support creativity. Having run a simulation, can users save the whole session and replay it later to study their performance or discuss it with a peer or mentor? Can they send the session to someone by email, annotate steps, or search for key events or actions? Can cxcellent sessions be stored in a digital library to allow future researchers or problem-solvers to build on the best work? 
Composing Artifacts and Performances

Another large family of software tools already supports creation of artifacts and performances. The ubiquitous word processor is the premier example of a flexible tool that enables many users to create diverse high quality printed documents using a relatively low cost laser or ink jet printer. Only a few decades ago such high production quality required advanced typesetting skills that were available to only a small number of people in the publishing industry. The word processor enables business people to produce elegant newsletters and professional advertising. It allows individuals to produce high quality resumes and elaborate full-length books. But word processors do more than facilitate quality appearance, they can also contribute to improved contents. The capacity to easily cut-and-paste, change terminology, or add references enables more people to create stronger scientific papers, legal briefs, or business proposals. It enables novelists, playwrights, and poets to easily accommodate suggestions from peers and mentors.

But even the word processor can be improved by thinking about creativity support in the genex framework. If new artifacts are inspired by previous work, then users should benefit from orderly processes for locating appropriate previous work and modifying it to suit their current needs. The basic notions of exemplars, templates, and macros, have already begun to appear. Authors of business letters can purchase thousands of exemplars that they can adapt in their own word processor. Then some of these authors might be able to contribute or sell their improved or specialized exemplars through growing digital libraries, available through the World-Wide Web.

A more flexible strategy is to prepare templates, as is done for business documents such as invoices or travel expense reports. These are supplied with many word processors. Templates differ from exemplars in that they are somewhat more flexible, may include computational capabilities, and can have instructional guidance. Macros extend the flexibility and allow richer transformations of documents.

Making a wider range of exemplars, templates, and macros available and a mechanism to create a market in new ones are still needed. Microsoft Office contains templates for word processed newsletters, slide presentations sales reports, spreadsheet invoices, photo library databases, etc. The structured processes of users's choices, called Wizards, have been improved to give an overview of the steps for tasks such as generating a personal resume or a legal pleading.

Similarly, Adobe PhotoDeluxe provides excellent visual guides for the complex processes in getting, cropping, editing, annotating, and sending images. Adobe Photoshop offers useful macros for redoing images to give them the appearance of Van Gogh's bold brush strokes, Seurat pointillism, or other styles. Music composition programs also provide macros to transform a phrase or motif and generate New Orleans jazz or Rhythm and Blues pieces (www.pgmusic.com). Templates for fugues or sonatas and the capacity to recast motifs in the style of Brahms, Beethoven or other composers seem realizable.

\section{Reviewing and Replaying Session Histories}

Reflecting on work is a central notion in quality improvement, creativity, and education methods. The metacognitive processes that promote self-awareness are learnable, but software support to capture the history of all user actions would seem to be a good foundation for many services. We now expect that web browsers record the history of our site visits, so that we can return to them. One study of World-Wide Web users showed that $58 \%$ of all URLs had been previously visited [21]. Even in this simple case controversies abound because the strategy for producing a compact, meaningful list (in a linear, tree, or network format) is not apparent. Another problem is that web sites may change over time, so older histories may no longer produce the same results.

Producing histories of command lines in Unix or information retrieval programs is relatively easy, but understanding the meaning of each command may depend on the context (for example, the current directory). In richer graphical user interface environments such as simulators, image manipulators, legal information retricval, or gcographic information systems, recording each user step is feasible. However, success requires careful user interface and software design to ensure that the results are compact, comprehensible, and useful. Once these basics are accomplished, users can manipulate session histories and replay the steps on another set of data or go back to change a step before replaying. Saving a sequences of steps for later use creates a basic macro, and by adding conditional and looping constructs quite ambitious programs could be built. For example, in a simulation of computer chip fabrication, it should be possible to rerun the simulation changing the temperature by single degree increments from 100 to 200 degrees Centigrade. If histories are first class objects then users should be able to send them to peers or mentors for comment or assistance with problems. Histories might also be searchable so that a set of hundreds of directories could be searched to see if any were done at a temperature above 200 degrees Centigrade. Users should be able to posî or sell exemplars of excellence or macros that might be helpful to others.

\section{Disseminating Results}

Email, listservs, digital libraries, the World Wide Web provide an excellent foundation for disseminating results but these also could be improved by thinking of the genex framework. The first circle of people to announce an innovation to would be previous and current workers. While digital libraries and online resources may make previous work accessible, extracting email (or mailing) addresses and ensuring that they are current is tedious, at best. Finding current workers in a domain is sometimes 
possible by listservs, newsletters, or websites of relevant communities.

A second circle of interest might be readers of papers or viewers of websites with related materials. Finding the identity of website viewers is not usually possible, but registration strategies might be developed to enable visitors to request information about future developments. The commercial bookseller amazon.com offers a service that will send you email when books on a topic or by a given author appear. Obviously, such registration lists have great commercial value, but publicly available variants are likely to emerge and be appealing because of their narrow focus. These are modern versions of what information retrieval professionals call selective dissemination of information (SDI), a policy by which users would be informed on a regular basis about new publications on topics they have selected.

Digital libraries could be reconceived of as digital library communities, by extensive use of online community software to turn every object into the focus of a discussion group. Anyone retrieving a novel, scientific paper, legal precedent, or classical music score could register to participate in a discussion of that item. Such online discussion groups would be a great stimulant to creative work in many disciplines. Additions to scientific journals, music libraries, or art galleries would require review by editors, collections managers, and curators. Modem technologies not only speed up the process, but also facilitate review from multiple sources. Maybe more importantly the complexity and cost of establishing online journals, libraries, and galleries is substantially less than physical institutions, thereby lowering the barrier. Having more diverse institutions that create digital libraries is also likely to stimulate creativity.

\section{CONCLUSIONS}

Ambitious visions can be helpful in shaping more concrete research agendas. For example, Engelbart's goal of augmenting human intellect [22] led to innovations such as the mouse and windows. Later, Brooks's belief in the importance of toolmaking [23] led to innovations such as haptic feedback in 3-d graphical environments. These inspirational visions were important catalysts for genex. The ambition to support creativity led to the four phases of genex.These four phases and the eight activities described in this paper are major challenges but the integrated combination of them could produce an environment that greatly facilitates creativity. The eight activities and their integration form a research agenda for human-computer interaction and user interface design.

The goal of supporting more creativity by more people more of the time is attractive, but there is the danger that the genex framework might be ineffective or even limit creativity. By making easy access to previous work and current workers, there is a risk that more exotic ideas will be suppressed. Similarly, using creativity supports such as simulations and composition tools may restrict imagination to only what is possible with these tools. Consultations are time consuming, and discouraging advice for novel ideas is a possible outcome. Fear that others will plagiarize compositions or steal inventions is another legitimate concem. An understanding of the dangers is important in pursuing the positive possibilities.

Between the lofty ambitions and troubling fears, lies the practical path of careful research and detailed design for the eight activities described in this paper. They need development, testing, and refinement to making them successful, find their flaws, and pursue alternatives. At every stage, widespread participation in design reviews increases the possibility that the resulting technologies will serve human needs in constructive and positive ways.

\section{ACKNOWLEDGEMENTS}

I am grateful for the thoughtful comments from Harry Hochheiser, Ross Malaga, Kent Norman, Catherine Plaisant, Jenny Preece, and Richard Salter. This work was supported in part by IBM Research's University Partner Program.

\section{REFERENCES}

1. Shneiderman, B. Codex, memex, genex: The pursuit of transformational technologies. International Journal of Human-Computer Interaction 10, 2 (1998), 87-106.

2. Csikszentmihalyi, M. Creativity: Flow and the Psychology of Discovery and Invention, HarperCollins, New York, 1996.

3. Boden, M. The Creative Mind: Myths \&Mechanisms. Basic Books, New York, 1990.

4. Cave, C. Creativity Web Site : http://www.ozemail.com.au/\%7Ecaveman/Creative/ind x.html (1999)

5. Couger, D. Creativity \& Innovation in Information Systems Organisations. Boyd \& Fraser Publishing. Co, Danvers, MA, 1996

6. Berners-Lee, T. et al. The World Wide Web. Communications of the ACM 37, 8 (1994), 76-82.

7. Shneiderman, B. Designing the user interface: strategies for effective human-computer interaction: third edition. Addison Wesley, Reading, MA, 1998.

8. North, C. \& Shneiderman, B. Snap-Together Visualizations: Co-ordinating multiple views to explore information. University of Maryland Dept of Computer Science Technical Report CS-TR4027 (1997).

9. Marchionini, G. Information Seeking in Electronic Environments, Cambridge University Press, UK, 1995. 
10. Dorcey, T. CU-SeeMe desktop videoconferencing software, Connexions 9,3 (1995). Also at http://cuseeme.cornell.edu/DorceyConnexions.html

11. Olsen, G.M. \& Olsen, J.S. Research on computersupported co-operative work, in Helander, M.G., Landauer, T.K. \& Prabhu, P.V. (eds), Handbook of Human-Computer Interaction: Second Edition, Elsevier, Amsterdam (1997), 1433-1456.

12. National Research Council - Committee on a National Collaboratory. National collaboratories: applying information technology for scientific research. National Academy Press, Washington, DC, 1993

13. Ahlberg, C \& Shnciderman, B. Visual information seeking: tight coupling of dynamic query filters with starfield displays. Proceedings of CHI'94, ACM Press, New York, NY (1994), 313-321 + color plates.

14. Bush, V. As we may think. Atlantic Monthly 76, 1 (1945), 101-108. Also at http://www2/the $\Lambda$ tlantic.com/atlantic/atlweb/flashbks/computer/tech.hm

15. Plaisant, C., Shneiderman, B. et al. Lifelines: visualizing personal histories. Proceedings of $\mathrm{CHI}$ '96, ACM Press, New York, NY (1996), 221-227, 518.
16.Roth, S.F., Lucas, P. et al. A user interface environment for exploring information. Proceedings Information Visualization '96, IEEE, Los Alamitos, CA (1996), 3-12.

17. Mayer,R.E. Thinking, Problem-Solving, Cognition, $2^{\text {nd }}$ Edition. W.H. Freeman, New York, NY (1992).

18. Massetti, B. An empirical examination of the value of creativity support systems on idea generation. MIS Quarterly 20, 1 (1996), 83-97.

19.Elam, J.J. \& Melissa, M. Can software influence creativity? Information Systems Research 1, (1990), 122.

20.Rose, A., Eckard, D. \& Rubloff, G. An application framework for creating simulation-based learning environments, University of Maryland Dept of Compute Science Technical Report CS-TR 3907 (1998).

21. Tauscher, L \& Greenberg, S. How people re-visit web pages: empirical findings and implications for the design of history systems. International Journal of Human-Computer Studies. Academic Prress, 1997. 